\title{
EFEITOS DE INSTRUÇÕES PRELIMINARES, ESPECÍFICAS E MÍNIMA SOBRE UMA TAREFA EXPERIMENTAL E SEGUIMENTO DE INSTRUÇÕES ${ }^{1}$
}

\author{
EFFECTS OF GENERAL, SPECIFIC AND MINIMAL INSTRUCTIONS IN AN \\ EXPERIMENTAL TASK AND INSTRUCTION FOLLOWING
}

\author{
Ronaldo Rodrigues Teixeira Júnior e Carla Cristina Paiva Paracampo \\ UNIVERSIDADE FEDERAL DO PARÁ
}

\begin{abstract}
RESUMO
Estudos sobre regras têm mostrado que alterações em trechos de diferentes tipos de instruções podem interferir no desempenho de participantes de pesquisa. O presente trabalho teve como objetivo avaliar os efeitos da apresentação de instruções preliminares, específicas e mínima sobre a realização de uma tarefa experimental e seguimento de instruções. Seis estudantes universitários foram expostos a um procedimento de escolha de acordo com o modelo, fazendo-se uso de um delineamento de sujeito como seu próprio controle. Os participantes foram divididos em duas condições que diferiam quanto ao tipo de instrução específica apresentada (correspondente ou discrepante). Nas Fases 1 e 2, os participantes não recebiam nenhuma instrução preliminar e eram expostos a instruções mínimas e específicas, de acordo com sua condição. Nas Fases 3 e 4, os mesmos participantes eram expostos a fases idênticas às anteriores, mas após terem recebido instruções preliminares, que incluíam descrições da tarefa, materiais e consequências. Os resultados mostraram que o fornecimento de instruções preliminares favoreceu a realização da tarefa e o seguimento de instruções, principalmente entre participantes que receberam instruções correspondentes. Esses dados sugerem que novos trechos de instruções preliminares precisam ser investigados para conhecer seus efeitos.
\end{abstract}

Palavras-chave: instruções preliminares, instruções específicas, instrução mínima, escolha de acordo com o modelo, estudantes universitários.

\begin{abstract}
Studies about rules have shown that changes in sections of different types of instructions may interfere in performance of research's subjects. The present work aimed to evaluate the effects of presentation of general, specific and minimal instructions in learning task and instruction following. Six college students were exposed to a maching-to-sample procedure, using a subject as his own control design. Subjects were divided in two conditions that differed as the type of specific instruction presented (accurate or inaccurate). In Phases 1 and 2, subjects received no general instructions and were exposed to minimal and specific instructions, according to their condition. In Phases 3 and 4, the same subjects were exposed to the same phases as before, but after receiving general instructions, which included passages about task, materials and consequences. Results showed that providing general instructions improved learning task and instruction following, especially among subjects who received accurate instructions. These data suggest that new sections of general instructions need to be investigated to know their effects.
\end{abstract}

Keywords: general instructions, specific instructions, minimal instruction, matching-to-sample, college students.

Quando pesquisas são realizadas com humanos, seus participantes recebem um número determinado de informações que permitem que eles entrem em contato com a situação experimental e executem alguma ação planejada pelo experimentador. Apesar de haver certa padronização dessas informações rece- bidas entre os participantes de um mesmo estudo, é comum observar muitas variações nas instruções elaboradas por experimentadores de grupos de pesquisa distintos (Pilgrim \& Johnston, 1988; Teixeira Júnior, 2009).

Além das instruções específicas, que incluem a descrição precisa de uma resposta

\footnotetext{
${ }^{1}$ Este trabalho se baseia no Experimento 1 da tese de doutorado do primeiro autor apresentada ao Programa de Pós-Graduação em Teoria e Pesquisa do Comportamento da Universidade Federal do Pará sob orientação da segunda autora e financiamento do CNPq, por meio de bolsa de doutorado para o primeiro autor (Processo nำ 142291/2007-2).
} 
que o participante deve emitir (e.g., aponte na sequência cor, forma, espessura; quando a luz verde acender, aperte o botão da esquerda e, quando a luz vermelha acender, aperte o botão da direita), outras instruções, chamadas de "gerais" ou "preliminares", com orientações básicas sobre o estudo (equipamentos, materiais, consequências etc.), costumam ser fornecidas pelos experimentadores antes do início dos procedimentos experimentais (cf. Albuquerque, Souza, Matos, \& Paracampo, 2003; Hayes, Brownstein, Zettle, Rosenfarb, \& Korn, 1986; Martinez \& Ribes, 1996; Newman, Buffington, \& Hemmes, 1995; Paracampo, Souza, Matos, \& Albuquerque, 2001). Nem sempre essas duas instruções são apresentadas isoladamente aos participantes de pesquisas, mas, em geral, os estudos na área de regras têm investigado os efeitos que manipulações em trechos de instruções específicas produzem no desempenho dos participantes. Entretanto, alguns estudos experimentais também têm mostrado que alterações em outros trechos de instruções, que não especificam respostas a serem emitidas, também podem interferir nos resultados.

Weiner (1970), por exemplo, observou que, quando participantes recebiam instruções com o número máximo de reforçadores que poderiam ser obtidos no estudo, eles apresentavam número menor de respostas em fases de extinção do que participantes que não recebiam essas instruções ou recebiam instruções dizendo que o número de reforçadores disponíveis era maior do que o que poderia ser obtido. Martinez e Tamayo (2005) observaram que, quando os participantes tinham de escolher entre estímulos diferentes, similares ou iguais ao modelo, a sim- ples escolha da expressão "mais semelhante" no lugar da palavra "similar" nas instruções era suficiente para que os participantes escolhessem estímulos iguais, e não similares, ao modelo, afetando, assim, a eficácia dos desempenhos obtidos em seu estudo.

Outros estudos aprofundaram essa investigação e podem ser descritos em mais detalhes. DeGrandpre, Buskist e Cush (1990) procuraram investigar os efeitos de instruções que descreviam a tarefa experimental e o equipamento (que eles chamaram de "instruções de orientação”) sobre o desempenho de participantes expostos a um esquema múltiplo de reforçamento. Eles distribuíram 20 estudantes universitários em quatro grupos, e cada grupo foi exposto a um conjunto diferente de instruções de orientação. Os participantes deveriam apertar a tecla de um computador de acordo com um esquema múltiplo de intervalo fixo (FI) e razão fixa (FR). Os resultados mostraram que os participantes que receberam as instruções mais detalhadas, as quais especificavam a tarefa, o botão para resposta e que deveriam ganhar o máximo de pontos que conseguissem, apresentaram a menor variabilidade de respostas e a menor sensibilidade às contingências programadas em comparação aos participantes dos outros três grupos. Os participantes que receberam as mesmas instruções, mas não foram informados de que deveriam ganhar o máximo de pontos que conseguissem, também apresentaram pouca variabilidade de respostas, mas apresentaram a maior sensibilidade às contingências programadas em relação aos participantes dos outros três grupos. Os participantes que receberam instruções que não especificavam a tarefa, ou do grupo que a instrução não especificava o botão 
para a resposta, apresentaram a maior variabilidade de respostas em comparação aos participantes dos outros dois grupos e pouca sensibilidade às contingências programadas. Os autores concluíram que as instruções de orientação afetam o contato dos participantes com as contingências. Segundo eles, esse tipo de instrução seria tão importante de ser investigada quanto outras instruções que têm sido investigadas em estudos da área.

Outro estudo nessa mesma direção foi o de Simonassi, Oliveira e Gosh (1997). O objetivo do Experimento 1 foi verificar se instruções que descrevem os termos da contingência programada afetam o comportamento de formular regras e solucionar um problema. Vinte estudantes universitários foram distribuídos em duas condições experimentais. Em uma das condições, a instrução especificava os termos da contingência programada (escreva nesta folha como você está distribuindo os cartões com números em relação às caixas verde e vermelha); na outra, era fornecida uma instrução genérica (escreva nesta folha como você está fazendo para resolver o problema). Nas duas condições, a tarefa era colocar um de vários cartões numerados em uma de duas caixas de cores diferentes. As respostas corretas eram colocar cartões com números pares em uma caixa verde e cartões com números ímpares em uma caixa vermelha. Em alguns intervalos entre as tentativas, o participante era solicitado a descrever como estava resolvendo o problema. Os resultados mostraram que nove dos 10 participantes de cada condição resolveram o problema. Dos nove participantes de cada condição que resolveram o problema, oito formularam a regra na condição de instrução específica e seis formularam a regra na condição de instrução genérica. Entre aqueles que formularam a regra, cinco dos oito participantes o fizeram até a terceira tentativa na condição de instrução específica, enquanto, na condição de instrução genérica, apenas um dos seis participantes fez o mesmo. Além de mais participantes da condição de instrução específica terem formulado regras e feito isso até a terceira tentativa, os participantes dessa condição também usaram com mais frequência palavras utilizadas na instrução do que os participantes da condição de instrução genérica. Os autores concluíram que a solução do problema dependeu das contingências programadas nas duas condições, enquanto a formulação da regra foi afetada pelo tipo de instrução que foi fornecido aos participantes.

Seguindo essa mesma linha, England e Buskist (1995) avaliaram a efetividade do controle instrucional quando os participantes são solicitados a não revelar informações sobre a tarefa experimental. No Experimento 1,18 estudantes universitárias receberam instruções detalhadas, gerais ou nenhuma instrução sobre discutir detalhes do experimento com outros participantes. A tarefa das participantes era apertar botões em um procedimento de escolha de acordo com o modelo. Após a conclusão da última sessão, as participantes eram colocadas com outras pessoas em uma sala, que perguntavam informações sobre a tarefa experimental ("cúmplices" do experimentador). Os resultados mostraram que, quando as participantes receberam instruções detalhadas, pedindo para que não conversassem com outros participantes e que isso poderia afetar o experimento, cinco das seis participantes não revelaram informações relevantes sobre a tarefa experimental; quan- 
do as participantes receberam instruções gerais, pedindo apenas para que não conversassem com outros participantes, ou quando as participantes não receberam qualquer instrução a esse respeito, cinco das seis participantes de cada grupo revelaram todos os detalhes da tarefa experimental. Os autores concluíram que, quando as instruções informavam as consequências negativas de não segui-la, elas foram mais efetivas para controlar o comportamento das participantes. Por outro lado, quando as instruções não informavam essas consequências, elas foram ineficientes em estabelecer o comportamento solicitado. Isso ocorreu independentemente de haver ou não o pedido nas instruções para que as participantes não falassem sobre a tarefa, o que sugeriria que instruções que não contêm trechos sobre as consequências de não segui-la podem ter o mesmo efeito que a ausência do fornecimento de qualquer instrução.

Os resultados desses estudos apontam para uma relação entre a manipulação de trechos distintos das instruções, a instalação e a manutenção do comportamento de seguir instruções e o controle pelas contingências. Em DeGrandpre et al. (1990), manipulações em trechos das instruções sobre o equipamento e a tarefa afetaram o controle pelos esquemas de reforçamento; em Simonassi et al. (1997), trechos das instruções que descreviam termos das contingências também interferiram no desempenho dos participantes; em England e Buskist (1995), trechos com informações sobre as consequências de não seguir a instrução afetaram seu seguimento.

Contudo, dentre os estudos citados, apenas o conduzido por DeGrandpre et al. (1990) manipulou diretamente trechos das instruções preliminares que descreviam a tarefa experimental e o equipamento utilizado no estudo, com o objetivo de avaliar os efeitos dessas manipulações sobre o desempenho dos participantes em um esquema múltiplo. Considerando-se a relevância desse tipo de manipulação para a identificação das variáveis que interferem tanto na execução correta da tarefa experimental quanto no controle por instruções específicas apresentadas subsequentemente aos participantes ou no controle pelas contingências de reforço programadas, o presente estudo pretendeu dar continuidade a essa linha de investigação. Nesse sentido, o presente estudo teve como objetivo avaliar e comparar os efeitos da apresentação de uma instrução mínima isolada com os efeitos da apresentação de instruções preliminares seguida da apresentação de uma instrução mínima sobre a execução da tarefa experimental (ou topografia de respostas emitidas) e o seguimento subsequente de instruções específicas.

Mais especificamente, pretendeu avaliar: a) os efeitos da apresentação de uma instrução mínima (que descreve apenas a resposta a ser emitida - apontar em sequência para três objetos) sobre a execução da tarefa experimental; b) os efeitos da apresentação de instruções preliminares (que descrevem detalhadamente características dos materiais utilizados no estudo, a resposta a ser emitida e consequências da emissão dessa resposta) sobre a execução da tarefa experimental; e c) os efeitos da apresentação de instruções específicas correspondentes ou discrepantes das contingências programadas (que descrevem a resposta específica a ser emitida - apontar em uma determinada sequência para três objetos) sobre o seguimento de instruções; 
expondo os participantes a um procedimento de escolha segundo o modelo similar ao adotado por Albuquerque et al. (2003) e utilizando um delineamento de sujeito como seu próprio controle.

Os resultados dessas manipulações permitirão identificar quais tipos de instruções (se mínima e/ou preliminares) utilizadas em situações experimentais são necessários para a execução correta da tarefa experimental, em termos da topografia de respostas requerida, e para a ocorrência e/ou manutenção do comportamento subsequente de seguir instruções específicas correspondentes e discrepantes.

\section{MÉTODO}

\section{Participantes}

Foram selecionados seis estudantes universitários, de diferentes cursos (exceto o de Psicologia), matriculados em diferentes semestres. O convite foi feito abordando aleatoriamente estudantes nos corredores de uma universidade pública federal. Todos foram convidados da seguinte forma: "Por favor, trabalho no laboratório de Psicologia da Universidade e estamos precisando de alunos de diferentes cursos para participar de uma pesquisa. Você teria interesse em participar? O objetivo dessa pesquisa é investigar processos básicos de aprendizagem em que as pessoas devem apenas apontar para objetos em uma mesa. A pesquisa será realizada em um único dia, aqui mesmo na universidade, e tem duração de aproximadamente 40 minutos. A pessoa que aceitar participar receberá a passagem de ônibus de ida e volta à universidade, além de poder receber mais uma quantia em dinheiro no final da pesquisa."

\section{Equipamento e material}

Foi utilizada uma mesa de madeira, medindo $150 \times 78 \times 70 \mathrm{~cm}$. Fixado à mesa, de modo a dividi-la ao meio em todo o seu comprimento, havia um anteparo com espelho unidirecional de $150 \times 60 \mathrm{~cm}$, fixado em uma moldura de madeira e localizado $13 \mathrm{~cm}$ acima do tampo da mesa. No centro do anteparo, junto ao tampo da mesa, havia uma abertura retangular de $45 \times 3 \mathrm{~cm}$. Dois centímetros acima e ao centro dessa abertura havia um contador operado pelo experimentador e com os dígitos voltados para o participante. Uma lâmpada fluorescente de 15 watts estava instalada na borda superior ao centro do anteparo. A mesa estava situada no centro de uma sala climatizada e com baixa iluminação.

Os estímulos modelo e de comparação foram 160 peças de madeira, partes de quatro conjuntos iguais de blocos lógicos (marca Funbec), variando em três dimensões: forma (quadrado, círculo, retângulo e triângulo); cor (azul, vermelha e amarela); e espessura (grossa e fina). Essas peças de madeira formavam 40 diferentes arranjos de estímulos, cada um constituído de um estímulo modelo e três estímulos de comparação. Cada estímulo de comparação apresentava apenas uma dimensão (cor, espessura ou forma) em comum com o estímulo modelo.

A combinação dos estímulos era aleatória, assim como a ordem de apresentação dos 40 arranjos. Os estímulos foram apresentados ao participante através da abertura retangular na base do anteparo divisor da mesa, em uma bandeja de madeira em forma de "T". Na parte final do cabo dessa bandeja, rente à base retangular, quatro ripas de madeira formavam um quadrado, no qual era 
colocado o estímulo modelo. Na base retangular da bandeja, dividida por ripas de madeira em três quadrados, foram apresentados os três estímulos de comparação. As respostas de escolha emitidas pelos participantes eram registradas pelo experimentador em um protocolo previamente preparado.

\section{Procedimento}

Durante as sessões experimentais, participante e experimentador ficavam sentados à mesa de frente um para o outro, separados pelo anteparo divisor da mesa. A lâmpada na borda superior do anteparo ficava constantemente acesa, voltada para o participante, de maneira a assegurar que seu lado apresentasse iluminação em maior intensidade e garantindo que apenas as ações emitidas pelo participante pudessem ser observadas através do espelho pelo experimentador. Os arranjos de estímulos previamente preparados ficavam sobre a mesa, ao lado do experimentador, na ordem em que eram apresentados.

Em cada tentativa, o experimentador apresentava um dos 40 arranjos de estímulos. $\mathrm{Na}$ presença desses estímulos, o participante devia apontar para cada um dos três estímulos de comparação, de acordo com o modelo, em uma dada sequência (sequência completa). Caso a sequência de respostas emitida estivesse de acordo com as contingências de reforço programadas (sequência correta), um ponto era acrescentado no contador e a bandeja com o arranjo de estímulos era retirada. Caso não houvesse nenhuma contingência de reforço programada durante a emissão de uma sequência de respostas ou a sequência emitida estivesse incorreta, a bandeja com o arranjo de estímulos era retirada sem que fossem acrescentados pontos no contador.
Caso o participante emitisse outras respostas que não fossem uma sequência completa, a bandeja era retirada após três segundos da emissão da última resposta, sem serem acrescentados pontos ao contador. O intervalo entre tentativas era de aproximadamente cinco segundos para a troca dos arranjos da bandeja e registro das respostas.

\section{Instruções}

$\mathrm{Na}$ primeira fase, quando participante e experimentador entravam na sala, a bandeja com um arranjo de estímulos ficava sobre a mesa, visível ao participante. $\mathrm{O}$ experimentador pedia ao participante para sentar-se na cadeira e ler o termo de consentimento.Após a assinatura do termo, e dependendo da fase experimental, o experimentador lia ou entregava ao participante, pela abertura na base do anteparo, uma folha de papel contendo uma das seguintes instruções (adaptadas de Albuquerque et al., 2003):

Instruções preliminares: "Este objeto, aqui em cima, é um modelo. Estes três objetos, logo abaixo, são para você comparar com o modelo. Observe que cada um dos objetos de comparação tem uma propriedade comum ao modelo.Veja, este só tem a espessura comum ao modelo; este só tem a cor comum ao modelo; e este só tem a forma comum ao modelo. Durante a pesquisa, você poderá ganhar pontos que serão trocados por dinheiro. Para ganhar pontos, você deverá apontar com o dedo em alguma sequência para cada um dos três objetos de comparação. Fazendo isso, você poderá ganhar pontos que serão mostrados no contador à sua frente.Veja como os pontos aparecem no contador (o experimentador acionava o contador três vezes). Cada 
ponto que ganhar depois será trocado por $\mathrm{R} \$ 0,10$ (10 centavos de real), mas apenas no final da pesquisa. Entendeu?"

Se apresentada, a instrução preliminar era lida apenas uma vez pelo experimentador, no início da fase. As dúvidas eram respondidas repetindo os trechos correspondentes presentes na instrução, quando fosse necessário.

Instrução mínima: Não descrevia nenhuma sequência de respostas, apenas repetia o trecho geral sobre a tarefa da instrução preliminar: "Quando eu mostrar estes objetos para você, aponte com o dedo em alguma sequência para cada um dos três objetos de comparação."

Instrução correspondente: Descrevia a sequência de respostas "CEF", 2 que correspondia às contingências programadas: "Quando eu mostrar estes objetos para você, primeiro aponte com o dedo para o objeto de comparação que tem a mesma cor do modelo; depois, para o objeto de comparação que tem a mesma espessura do modelo; e, em seguida, para o objeto de comparação que tem a mesma forma do modelo. Ou seja, você deve apontar, na sequência, cor, espessura e forma."

Instrução discrepante: Descrevia a sequência de respostas "FCE", 3 que não correspondia às contingências programadas: "Quando eu mostrar estes objetos para você, primeiro aponte com o dedo para o objeto de comparação que tem a mesma forma do modelo; depois, para o objeto de comparação que tem a mesma cor do modelo; e, em seguida,

\footnotetext{
${ }^{2}$ Nos casos em que o participante era exposto a outra fase de instrução correspondente, a sequência CEF foi substituída por FEC, para controle do efeito de história.

${ }^{3}$ Nos casos em que o participante era exposto a outra fase de instrução discrepante, a sequência FCE foi substituída por CFE, para controle do efeito de história.
}

para o objeto de comparação que tem a mesma espessura do modelo. Ou seja, você deve apontar, na sequência, forma, cor e espessura."

A instrução mínima e as instruções específicas (correspondente ou discrepante) eram apresentadas apenas uma vez, no início de cada fase experimental. Imediatamente após entregá-las ao participante, o experimentador dizia: "Leia estas instruções com atenção. Quando você achar que as entendeu, me devolva por esta abertura." No caso de dúvidas que não pudessem ser esclarecidas pela releitura da instrução, o experimentador dizia: "Não posso lhe fornecer nenhuma informação adicional. Pode fazer como tiver entendido." Logo após o participante devolver a folha com as instruções, o experimentador removia a bandeja, voltava a apresentá-la com um novo arranjo de estímulos e dizia: "Agora só posso falar com você no final desta sessão. Pode começar a apontar." Novas perguntas ou comentários durante a sessão eram ignorados ou, caso fosse necessário, era repetida alguma das frases padronizadas já faladas anteriormente.

\section{Delineamento experimental}

Os seis participantes primeiramente eram expostos a duas fases em que nenhuma instrução preliminar era apresentada e, em seguida, eram expostos a duas fases idênticas às duas primeiras, sendo a terceira fase iniciada com a apresentação da instrução preliminar.

Os participantes foram distribuídos em duas condições experimentais, (Condição Correspondente e Condição Discrepante). Cada uma das condições era composta de quatro fases (ver Tabela 1).

A Fase 1 das duas condições era iniciada com a apresentação da instrução mínima. A 
Tabela 1

Esquema do procedimento das Condições Correspondente e Discrepante

\begin{tabular}{lccccc}
\hline \multirow{2}{*}{ Condições } & Fases & Instruções & \multicolumn{2}{c}{ Sequência } & \multirow{2}{*}{ Esquema } \\
\cline { 3 - 5 } & 1 & Mínima & - & - & - \\
Correspondente & 2 & Correspondente & CEF & CEF & CRF \\
& 3 & Preliminar + Mínima & - & Reforçada & \\
\hline & 4 & Correspondente & FEC & FEC & CRF \\
\hline \multirow{2}{*}{ Discrepante } & 1 & Mínima & - & - & - \\
& 2 & Discrepante & FCE & CEF & CRF \\
& 3 & Preliminar + Mínima & - & - & - \\
& 4 & Discrepante & CFE & EFC & CRF \\
\hline
\end{tabular}

Fase 2 da Condição Correspondente era iniciada com a apresentação da instrução correspondente, e a Fase 2 da Condição Discrepante, com a apresentação da instrução discrepante. A Fase 3 das duas condições era iniciada com a apresentação da instrução preliminar seguida pela apresentação da instrução mínima. A Fase 4 da Condição Correspondente era iniciada com a apresentação da instrução correspondente, e a Fase 4 da Condição Discrepante, com a apresentação da instrução discrepante.

Nas duas condições, as Fases 1 e 3 eram constituídas por 10 tentativas de linha de base. Essas fases tiveram como objetivo avaliar e comparar os efeitos da apresentação da instrução mínima isolados dos efeitos da apresentação de instruções preliminares seguida da apresentação da instrução mínima sobre a topografia de respostas emitidas pelos participantes. Durante as Fases 1 e 3 das duas condições, nenhuma resposta era reforçada. Na Fase 2 da Condição Correspondente, era reforçada, em CRF, a sequência CEF, e, na Fase 4, a sequência FEC (sequências instruídas). Na Fase 2 da Condição Discrepante, era reforçada, em CRF, a sequência CEF, e, na Fase 4, a sequência EFC (sequências diferentes das instruídas). Caso algum participante emitisse nas Fases 1 e 3, em mais de 50\% das tentativas, alguma das sequências que seriam instruídas ou programadas para produzir reforços nas Fases 2 e 4, ele não era exposto às fases seguintes e sua participação no estudo era encerrada. Esse procedimento foi adotado para evitar coincidência entre o comportamento apresentado pelo participante nas Fases 1 e 3 e o comportamento descrito na instrução nas Fases 2 e 4.

As Fases 1 e 3 eram encerradas após 10 tentativas serem apresentadas, e as Fases 2 e 4, das duas condições, eram encerradas após a 
ocorrência de 30 tentativas ou após 10 tentativas seguidas sem a emissão de uma sequência completa pelo participante. A transição entre as fases era marcada pela apresentação de uma nova instrução e por mudanças nas contingências. As sessões eram realizadas em um único dia, com duração aproximada de 40 minutos.

\section{Resultados}

De modo geral, observou-se grande variabilidade na topografia de respostas dos participantes em relação à execução da tarefa descrita. Em função disso, foram criadas as seguintes categorias de topografia de respostas: A - apontar com o dedo em sequência para os três objetos de comparação (correspondência precisa); B - apontar com a mão ou levantar em sequência os três objetos de comparação (correspondência parcial); C - apontar ou levantar os três objetos de comparação, incluindo o modelo antes, durante ou depois da sequência de respostas (correspondência parcial); D - apontar ou levantar apenas um ou dois objetos de comparação por tentativa (sem correspondência); E - trocar peças de lugar ou levantá-las de forma aleatória (sem correspondência). $\mathrm{O}$ uso de uma categoria de topografia de respostas indica que o participante emitiu a resposta descrita em pelo menos $60 \%$ das tentativas da fase.

A Tabela 2 mostra a topografia de respostas nas quatro fases e o número de emissões das sequências instruídas (ESI) nas Fases 2 e 4 emitidas por cada participante das duas condições. Considerou-se que um participante seguiu instruções se ele emitisse a sequência instruída em pelo menos $70 \%$ das tentativas, ou seja, em no mínimo 21 das 30 tentativas das Fases 2 ou 4.

Tabela 2

Topografia de respostas nas quatro fases e o número de emissões das sequências instruídas (ESI) nas

Fases 2 e 4 emitidas por cada participante das duas condições

\begin{tabular}{ccccc}
\hline $\begin{array}{c}\text { Participante/ } \\
\text { condição }\end{array}$ & Fase 1 & Fase 2 & Fase 3 & Fase 4 \\
\cline { 2 - 5 } P01/corresp. & Topografia & Topografia/ESI & Topografia & Topografia/ESI \\
P02/corresp. & E & E 0 tentativa & D & A 27 tentativas \\
P03/corresp. & E & A 23 tentativas & A & A 30 tentativas \\
\hline P04/discrep. & E & E 0 tentativa & A & A 30 tentativas \\
P05/discrep. & E & E 0 tentativa & C & D 5 tentativas \\
P06/discrep. & C & D 3 tentativas & E & E 0 tentativa \\
\hline
\end{tabular}

Nota: A letra A indica que a topografia da resposta correspondeu precisamente à tarefa fornecida pelo experimentador, enquanto as letras B e $\mathrm{C}$ indicam que correspondeu parcialmente. As letras D e E indicam que não houve correspondência. 
Pode-se observar que, na Fase 1, iniciada com a apresentação da instrução mínima, sem apresentação prévia de instrução preliminar, apenas um dos seis participantes realizou a tarefa de forma correspondente à descrita (P06). Os demais apresentaram respostas aleatórias, trocando peças de lugar ou levantando-as sem nenhuma correspondência com a tarefa (Categoria E).

$\mathrm{Na}$ Fase 2, em que instruções correspondentes ou discrepantes eram fornecidas, apenas P02 realizou a tarefa de forma precisa (apontou em sequência para os três objetos de comparação) e seguiu a instrução correspondente em 23 das 30 tentativas da fase. Os demais participantes mantiveram a emissão de respostas aleatórias (Categoria E) ou apontaram para apenas um dos objetos de comparação (Categoria D), inclusive P06, que na fase anterior tinha sido o único a responder de forma correspondente à tarefa descrita.

$\mathrm{Na}$ Fase 3, iniciada com a apresentação de instruções preliminares, com apresentação posterior da instrução mínima, quatro dos

Sem Instrução Preliminar

(Fase 1)

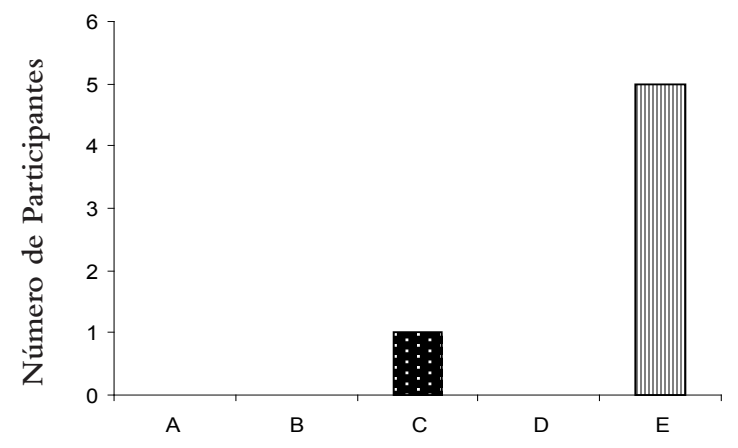

Categorias de Desempenho seis participantes (dois da Condição Correspondente e dois da Condição Discrepante) realizaram a tarefa de forma correspondente à descrita (P02, P03 e P06 emitindo respostas da Categoria A e P04, da Categoria C).

$\mathrm{Na}$ Fase 4, em que instruções correspondentes e discrepantes eram fornecidas, quatro dos seis participantes realizaram a tarefa de forma precisa (Categoria A) e também seguiram instruções na maior parte das tentativas. Entretanto, desses quatro, três (P01, P02 e P03) eram da Condição Correspondente e apenas um (P06), da Condição Discrepante.

A Figura 1 mostra o número de participantes e os tipos de topografia de respostas emitidas nas duas condições, durante as fases de instrução mínima. Observa-se que, na Fase 1 (sem o fornecimento da instrução preliminar), cinco dos seis participantes emitiram respostas sem correspondência com a tarefa (Categoria E), enquanto, na Fase 3 (com o fornecimento da instrução preliminar), quatro dos seis participantes emitiram respostas com correspondência precisa ou parcial à tarefa (Categorias A e C).
Com Instrução Preliminar

(Fase 3)

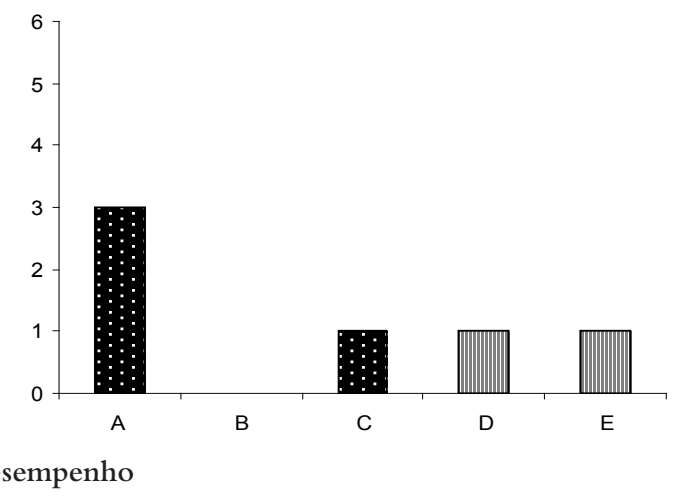

Figura 1. Topografia de respostas emitidas pelos participantes das duas condições nas Fases 1 e 3. As categorias A, B e C se referem às respostas com correspondência precisa ou parcial com a tarefa (preto pontilhado), e as categorias D e E se referem às respostas sem correspondência com a tarefa (cinza vertical). 
A Figura 2 mostra o número de participantes das Condições Correspondente e Discrepante que seguiram as instruções apresentadas nas Fases 2 e 4. Observa-se que, na Fase 2 (antes da exposição às instruções preliminares), apenas um participante da Condição Correspondente seguiu instruções, enquanto, na Fase 4 (após a exposição às instruções preliminares na Fase 3), todos os participantes da Condição Correspondente e apenas um da Condição Discrepante seguiram instruções.

Antes da Instrução Preliminar (Fase 2)

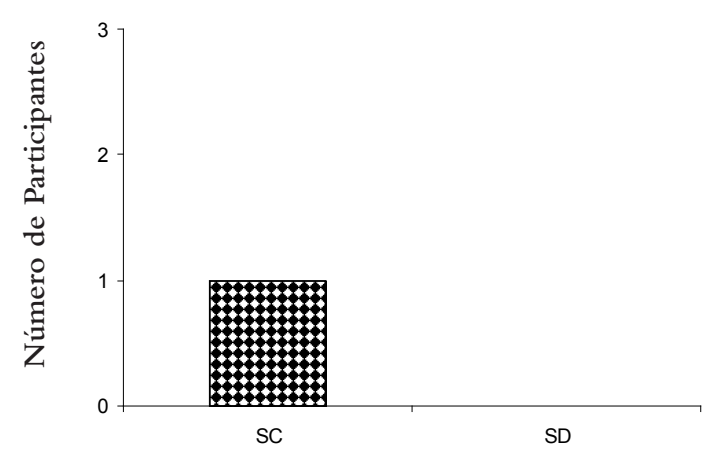

dessas fases. Durante as Fases 1 e 3 deste estudo, nenhum participante teve sua participação encerrada por emitir, em mais de 50\% das tentativas, alguma das sequências de respostas que seriam instruídas ou programadas para produzir reforços.

\section{Discussão}

Os resultados da Fase 1 dos cinco participantes (P01, P02, P03, P04 e P05) que

\section{Após a Instrução Preliminar}

(Fase 4)

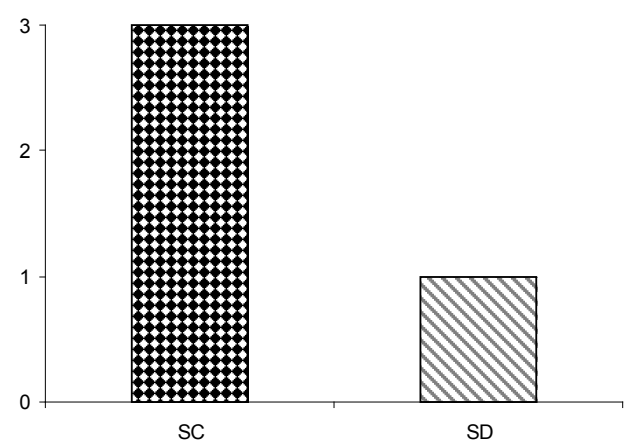

Seguimento de Instruções

Figura 2. Número de participantes das Condições Correspondente (preto xadrez) e Discrepante (cinza diagonal) que seguiram instruções correspondentes (SC) ou discrepantes (SD) nas Fases 2 e 4.

Neste estudo, só houve distribuição de pontos para os participantes da Condição Correspondente, pois nenhum participante da Condição Discrepante emitiu a sequência programada para produzir reforço nas Fases 2 e 4. Os participantes P01, P03, P04, P05 e P06 (durante a Fase 2) e P04 e P05 (durante a Fase 4) apresentaram desempenhos sem correspondência com tarefa (Categorias D ou E) durante 10 tentativas consecutivas e atingiram um dos critérios de encerramento emitiram topografias de respostas sem correspondência com a tarefa requerida, após a apresentação da instrução mínima, e os resultados da Fase 3 dos quatro participantes (P02, P03, P04 e P06) que emitiram topografias de respostas correspondentes à tarefa requerida, após a apresentação de instruções preliminares, mostram que a instrução preliminar foi uma variável relevante para a realização correta da tarefa experimental. A instrução mínima "aponte para os três objetos 
de comparação", a qual especificava apenas a resposta a ser emitida pelos participantes, não foi suficiente para instalar a topografia de respostas requerida para a execução correta da tarefa experimental em cinco dos seis participantes, indicando que informações presentes nas instruções preliminares a respeito de qual objeto era o modelo e quais objetos eram comparações, informações sobre as dimensões relevantes dos estímulos a serem consideradas para a emissão da resposta e informações sobre as consequências produzidas pela resposta são importantes para a realização precisa da tarefa experimental.

Contudo, vale destacar que P06 respondeu de forma parcialmente correspondente à tarefa requerida (Categoria C) na Fase 1, diferentemente dos demais participantes, que apresentaram padrões aleatórios de respostas (Categoria E) na Fase 1.É possível que P06, em sua historia pré-experimental, tenha adquirido alguma familiaridade ou habilidade específica com tarefas semelhantes à utilizada no presente estudo. Alguns estudos na área de controle por regras mostram como diferentes histórias pré-experimentais podem explicar diferenças importantes no desempenho de participantes em uma mesma tarefa experimental (Pinto, Paracampo, \& Albuquerque, 2006; Wulfert, Greenway, Farkas, Hayes, \& Dougher, 1994).

Além disso, não fica claro por que, mesmo após a apresentação de instruções preliminares, dois dos seis participantes (P01 e P05) não executaram corretamente a tarefa requerida, considerando que seus trechos descreviam claramente o que devia ser feito. É provável que a história prévia de exposição às Fases 1 e 2, nas quais não foram apresentadas instruções preliminares, possa ter mantido padrões de topografias de respostas sem correspondência com a tarefa, considerando que os participantes continuaram sendo expostos a novas tentativas durante o experimento, sem a interrupção ou correção do experimentador. Quando as instruções preliminares foram apresentadas, é possível que alguns participantes tenham mantido ou criado novos padrões de respostas sem correspondência com a tarefa requerida, porque seus desempenhos não produziram consequências diferenciais, em outras palavras, não produziram consequências explícitas, como, por exemplo, feedback para emissão de topografias de respostas corretas e incorretas ou consequências aversivas contingentes à emissão de topografias de respostas incorretas. A literatura na área de regras destaca como o uso de estímulos aversivos (perda de pontos) pode alterar a probabilidade do seguimento de instruções pelos participantes (Paracampo \& Albuquerque, 2004; Pinto, Paracampo, \& Albuquerque, 2008).

Esta análise sugere que outras variáveis, além das instruções preliminares, tais como diferenças individuais e história experimental, podem também ter interferido no desempenho dos participantes em termos da topografia de respostas emitidas.

Adicionalmente, é necessário considerar os efeitos da apresentação das instruções específicas sobre a execução correta da tarefa experimental. Os desempenhos de P02 na Fase 2 e de P01 na Fase 4 (ambos da Condição Correspondente) sugerem, no caso desses dois participantes, que as instruções específicas podem também ter favorecido a emissão de topografias de respostas corretas. Na Fase 2, após a apresentação da instrução específica correspondente, P02 deixou de emitir 
o desempenho sem correspondência com a tarefa que tinha emitido na Fase 1 (Categoria E) e passou a emitir desempenho com correspondência precisa à tarefa (Categoria A), seguindo as instruções correspondentes na maior parte das tentativas dessa fase. Do mesmo modo, na Fase 4, após a apresentação da instrução específica correspondente, P01 deixou de emitir o desempenho sem correspondência com a tarefa emitido nas fases anteriores (Categorias D e E) e passou a emitir desempenho com correspondência precisa à tarefa (Categoria A), seguindo as instruções correspondentes na maior parte das tentativas dessa fase.

As instruções específicas indicavam as sequências específicas de respostas que o participante deveria emitir; entretanto, seu conteúdo pode ter contribuído para a emissão de topografias corretas de respostas na medida em que descrevia as dimensões relevantes dos estímulos para a emissão da resposta, sugeria que os participantes deveriam relacionar os objetos de comparação com um modelo e descrevia precisamente os passos que o participante deveria seguir (“...primeiro, aponte com o dedo para o objeto de comparação que tem a mesma cor de modelo; depois...; em seguida...”). Contudo, os desempenhos de P06 na Fase 2 e de P04 na Fase 4 requerem que sejam considerados os efeitos de outras variáveis, além das características das instruções específicas, no estabelecimento e na manutenção da topografia correta de respostas e do comportamento de seguir instruções.

Os desempenhos de P06 na Fase 2 e de P04 na Fase 4 (ambos da Condição Discrepante), quando comparados com os desempenhos de P02 na Fase 2 e de P01 na Fase 4 (ambos da Condição Correspondente), suge- rem que o fato de a instrução específica descrever a sequência correta de respostas que produzia reforço na fase ou descrever outra sequência que não produzia reforço na fase também pode ter afetado a manutenção tanto da emissão da topografia correta de respostas quanto do comportamento de seguir instruções. Os resultados mostrando P06 (Condição Discrepante), que havia emitido desempenho correspondente à tarefa na Fase 1 e deixou de emitir esse desempenho na Fase 2 após três tentativas seguindo a instrução discrepante, e, similarmente, os resultados mostrando P04 (Condição Discrepante), que havia emitido desempenho correspondente à tarefa na Fase 3 e deixou de emitir esse desempenho na Fase 4 após cinco tentativas seguindo a instrução discrepante, apoiam essa sugestão. Ou seja, os desempenhos de P06 (Fase 2) e de P04 (Fase 4), bem como os desempenhos de P02 (Fase 2) e de P01 (Fase 4), sugerem que tanto a manutenção da topografia de respostas com correspondência precisa à tarefa quanto a manutenção do comportamento de seguir instruções dependeram, também, de se o seguimento da instrução produziu (instrução correspondente) ou não (instrução discrepante) reforçadores.

Em síntese, o resultado geral mostrando que mais participantes da Condição Correspondente realizaram a tarefa e seguiram as instruções quando comparados com participantes da Condição Discrepante apoia outros dados encontrados na literatura da área, que mostram que o seguimento de instruções tende a ser mantido quando estas correspondem às contingências programadas e, sob algumas condições, tende a ser abandonado quando as instruções não correspondem às 
contingências programadas (Albuquerque et al., 2003; Galizio, 1979; Hayes et al., 1986; LeFrançois, Chase, \& Joyce, 1988; Newman, Buffington, \& Hemmes, 1995; Paracampo et al., 2001; Ribes \& Martinez, 1990; Shimoff, Catania, \& Matthews, 1981).

Quanto aos efeitos da apresentação da instrução preliminar na realização da tarefa pelos participantes, os resultados do presente estudo sustentam a relevância desse tipo de investigação, de acordo com o que tem sido apontado na literatura (DeGrandpre et al., 1990; England \& Buskist, 1995; Martinez \& Tamayo, 2005; Simonassi et al., 1997; Weiner, 1970). A ausência de instruções preliminares descrevendo trechos sobre a tarefa, os materiais e as consequências programadas dificulta a realização da tarefa e mostra que os procedimentos que têm sido adotados, com o fornecimento de instruções preliminares, são importantes de ser mantidos nos estudos sobre regras, especialmente porque, conforme foi observado, sem a descrição precisa de como o participante deve realizar a tarefa, dificilmente se podem avaliar os efeitos de outras instruções fornecidas posteriormente.

Entretanto, não se sabe ao certo quais trechos das instruções preliminares são ou não essenciais para a realização da tarefa. Teixeira Júnior (2009), em um estudo teórico, comparou estudos de diferentes grupos de pesquisa e observou que os modelos de instruções fornecidas aos participantes variavam substancialmente. A esse respeito, Pilgrim e Johnston (1988) também chamam a atenção para a grande variedade de procedimentos que são utilizados em estudos que fazem uso de regras que muitas vezes não são acompanhados de análises, por parte dos experimentadores, de seus efeitos. O presente trabalho buscou contribuir nessa direção, comparando os efeitos de instruções preliminares, mínimas e específicas separadamente, em diferentes fases. Porém, outras investigações ainda precisam ser realizadas.

Por exemplo, com base apenas nos resultados do presente estudo, não se pode identificar qual dos trechos das instruções preliminares (tarefa, materiais e consequências), ou se todos eles, foi determinante para a ocorrência dos resultados observados. Considerando-se que o trecho que descrevia a tarefa experimental constava tanto das instruções preliminares quanto da instrução mínima, e que a maior parte dos participantes expostos somente à instrução mínima na Fase 1 não apresentou topografias de respostas correspondentes à tarefa experimental, fica a dúvida sobre qual dos outros trechos da instrução preliminar (materiais ou consequências) teria sido determinante para a emissão de topografias de respostas correspondentes à tarefa observadas no desempenho da maioria dos participantes na Fase 3. Talvez o trecho sobre os materiais favoreça a compreensão da tarefa, ou o trecho sobre as consequências favoreça o contato com as contingências, ou mesmo uma combinação de ambos. Estudos futuros poderiam isolar os efeitos de cada um desses trechos, o que permitiria conhecer melhor suas funções quando presentes em uma instrução preliminar.

Assim como os trechos sobre materiais e consequências, vários outros poderiam ser isolados e ter seus efeitos conhecidos. Trechos de saudação ou agradecimento, sobre a natureza ou o objetivo do estudo, demonstrações, exemplos etc. muitas vezes são acrescentados livremente às instruções fornecidas por experimentadores de diferentes grupos de pes- 
quisa, sem se saberem ao certo os efeitos que eles podem ter sobre o desempenho dos participantes. Novos estudos que manipulassem esses ou outros trechos de instruções preliminares poderiam contribuir para identificar precisamente quais trechos das instruções são essenciais para a realização da tarefa e o seguimento de regras, levando a um refinamento dos procedimentos utilizados tanto nessa área quanto em outros campos que utilizam humanos como participantes de pesquisa.

\section{REFERÊNCIAS}

Albuquerque, L. C., de Souza, D. G., Matos, M. A., \& Paracampo, C. C. P. (2003). Análise dos efeitos de histórias experimentais sobre o seguimento subsequente de regras. Acta Comportamentalia, 11, 87-126.

DeGrandpre, R., Buskist, W., \& Cush, D. (1990). Effects of orienting instructions on sensitivity to scheduled contingencies. Bulletin of Psychonomic Society, 28, 331-334.

England, D. E., \& Buskist, W. (1995). The effects of instructions on subjects disclosure of information about operant tasks. The Psychological Record, $45,451-461$.

Galizio, M. (1979). Contingency-shaped and rulegoverned behavior: Instructional control of human loss avoidance. Journal of the Experimental Analysis of Behavior, 31, 53-70.

Hayes, S. C., Brownstein, A. J., Zettle, R. D., Rosenfarb, I., \& Korn, Z. (1986). Rule governed behavior and sensitivity to changing consequences of responding. Journal of the Experimental Analysis of Behavior, 45, 237-256.

LeFrançois, J. R., Chase, P. N., \& Joyce, J. (1988). The effects of variety of instructions on human fixed-interval performance. Journal of the Experimental Analysis of Behavior, 49, 383-393.
Martinez, H., \& Ribes, E. (1996). Interactions of contingencies and instructional history on conditional discrimination. The Psychological Record, 46, 301-318.

Martinez, H., \& Tamayo, R. (2005). Interactions of contingencies, instructional accuracy, and instructional history in conditional discrimination. The Psychological Record, 55, 633-646.

Newman, B., Buffington, D. M., \& Hemmes, N. S. (1995). The effects of schedules of reinforcement on instruction following. The Psychological Record, 45, 463-476.

Paracampo, C. C. P., \& Albuquerque, L. C. (2004). Análise do papel das consequências programadas no seguimento de regras. Interação em Psicologia, 8, 237-245.

Paracampo, C. C. P., Souza, D. G., Matos, M. A., \& Albuquerque, L. C. (2001). Efeitos de mudança em contingências de reforço sobre o comportamento verbal e não-verbal. Acta Comportamentalia, 9, 31-55.

Pilgrim, C., \& Johnston, J. M. (1988). Laboratory lore and research practices in the experimental analysis of human behavior: Issues in instructing subjects. The Behavior Analyst, 11, 59-64.

Pinto, A. R., Paracampo, C. C. P., \& Albuquerque, L. C. (2006). Análise do controle por regras em participantes classificados de flexíveis e de inflexíveis. Acta Comportamentalia, 14, 171-194.

Pinto, A. R., Paracampo, C. C. P., \& Albuquerque, L. C. (2008). Efeitos de perda de reforçadores sobre o seguir regras em participantes flexíveis e inflexíveis. Revista Brasileira de Análise do Comportamento, 4, 111-125.

Ribes, E., \& Martinez, H. (1990). Interaction of contingencies and rule instructions in the performance of human subjects in conditional discrimination. The Psychological Record, 40, 565-586.

Shimoff, E., Catania, A. C., \& Matthews, B. A. (1981). Uninstructed human responding: Sensi- 
tivity of low-rate performance to schedule contingencies. Journal of the Experimental Analysis of Behavior, 36, 207-220.

Simonassi, L., Oliveira, C., \& Gosch, C. (1997). Exposição a contingências, conteúdo de instrução e formulação de regras. Psicologia: Teoria e Pesquisa, 13, 189-195.

Teixeira Júnior, R. R. (2009).Variáveis do comportamento governado por regras: uma análise de estudos da área. Acta Comportamentalia, 17, 351-385.

Weiner, H. (1970). Instructional control of human operant responding during extinction following fixed-ratio conditioning. Journal of the Experimental Analysis of Behavior, 13, 391-394.

Wulfert, E., Greenway, D. E., Farkas, P., Hayes, E. C., \& Douguer, M. J. (1994). Correlation between selfreported rigidity and rule-governed insensitivity to operant contingencies. Journal of the Experimental Analysis of Behavior, 27, 659-671.

Nota dos autores: Endereço para correspondência: Ronaldo Rodrigues Teixeira Júnior. Rua General Andrade Neves, 1.033/101, Grajaú, Belo Horizonte/MG, CEP: 30430-070. 